\title{
Lumen
}

Selected Proceedings from the Canadian Society for Eighteenth-Century Studies

\section{Le luxe, instrument de pouvoir dans l'Histoire de Madame la marquise de Pompadour traduite de l'anglais (1759) de Mlle de Falques}

\section{Isabelle Tremblay}

Volume 38, 2019

URI : https://id.erudit.org/iderudit/1059277ar

DOI : https://doi.org/10.7202/1059277ar

Aller au sommaire du numéro

Éditeur(s)

Canadian Society for Eighteenth-Century Studies / Société canadienne d'étude du dix-huitième siècle

ISSN

1209-3696 (imprimé)

1927-8284 (numérique)

Découvrir la revue

Citer cet article

Tremblay, I. (2019). Le luxe, instrument de pouvoir dans l'Histoire de Madame

la marquise de Pompadour traduite de l'anglais (1759) de Mlle de Falques.

Lumen, 38, 143-159. https://doi.org/10.7202/1059277ar 


\title{
Le luxe, instrument de pouvoir dans l'Histoire de Madame la marquise de Pompadour traduite de l'anglais (1759) de Mlle de Falques
}

\author{
ISABELLE TREMBLAY \\ Collège militaire royal du Canada
}

Si dans l'Europe française (1777), Louis-Antoine de Caraccioli soutient que «jadis tout était romain, aujourd'hui tout est français ${ }^{1}$ ", c'est que la France se démarque et s'impose au siècle des Lumières comme modèle à imiter ou du moins à envier. La politesse et le bon goût jouent un rôle important pour soutenir la réputation de ce royaume puissant. Le luxe et la mode, dont les défenseurs soutiennent qu'ils sont sources de prospérité, contribuent à leur tour à donner l'illusion que «Paris c'est le monde 2 », pour reprendre la réplique d'un personnage de Marivaux. Selon l'Encyclopédie, le luxe adoucit les mœurs, est favorable aux progrès des connaissances et des beaux-arts, augmente la puissance des nations, de même que le bonheur des citoyens ${ }^{3}$. Grâce à l'étendue et à l'éclat qu'il connaît en France, il contribue à légitimer et à magnifier le pouvoir établi, mais surtout à assurer à la France une place de choix au centre de l'Europe cosmopolite des Lumières. Joan Dejean soutient d'ailleurs qu'au XVIII ${ }^{\mathrm{e}}$ siècle, la «France avait acquis

1. Louis-Antoine Caraccioli, Paris, le modèle des nations étrangères ou l'Europe française, Paris, Veuve Duchesne, 1777, p. 3.

2. Pierre Carlet de Chamblain de Marivaux, La méprise, dans Euvres complètes de M. de Marivaux, Paris, Duchesne, 1781 [1734], t. III, p. 54.

3. Jean-François de Saint-Lambert, "Luxe», dans Encyclopédie ou Dictionnaire raisonné des sciences, des arts et des métiers par une Société de gens de lettres; mis en ordre et publié par M. Diderot et M. D’Alembert, Neuchâtel, Samuel Faulche, 1765, t. IX, p. $763-771$. 
une forme de monopole sur la culture, le style, le luxe, une position qu'elle continue d'occuper ${ }^{4}$ ». Sujet de débat économique, politique et moral, le luxe mobilise toutes les disciplines: histoire, politique, théologie, philosophie, morale, économie et littérature. Si l'imaginaire se présente comme le «support du luxe $e^{5}$ » à l'époque des grandes fêtes royales, à son tour, l'imaginaire du luxe tel qu'il se déploie dans la fiction des Lumières constitue un des supports du rayonnement de la France. C'est pourquoi la présente étude propose de s'interroger sur un texte fort controversé au moment de sa publication en raison de l'éclairage peu favorable qu'il réserve à la cour de Louis XV : l'Histoire de Madame la marquise de Pompadour traduite de l'anglais (1759) de Mlle de Falques.

Religieuse défroquée qui passe une grande partie de sa vie à Londres, préceptrice de William Jones junior, orientaliste célèbre, et des filles de Lady Elizabeth Craven ${ }^{6}$, secrétaire et copiste, Mlle de Falques, également connue sous les noms de Mlle de Fauque(s), de Mme de Vaucluse, de Mme de la Cépède(s) et de Mme Starck ${ }^{7}$, est romancière et conteuse $\mathrm{e}^{8}$. Comprise comme un «pamphlet parfois très violent ${ }^{9}$ » exposant les origines de la favorite de Louis XV, l'Histoire de Madame la marquise de Pompadour traduite de l'anglais que l'instance narrative désigne à l'aide du terme «brochure ${ }^{10}$ » et qu'un critique contemporain considère comme «la biographie la plus importante de

4. Joan Dejean, The Essence of Style: How the French Invented High Fashion, Fine Food, Chic, Cafés, Style, Sophistication, and Glamour, New York/London/ Toronto/Sydney, Free Press, 2005, p. 3. Je traduis.

5. Jean-Jacques Gautier, «L'imaginaire comme support du luxe: les fêtes royales de Versailles de mai 1664", dans Robert Fox et Anthony John Turner (dir.), Luxury Trades and Consumerism in Ancien Régime Paris: Studies in the History of the Skilled Workforce, Aldershot, Ashgate, 1998, p. 191-219.

6. Michael J. Franklin, Orientalist Jones: Sir William Jones, Poet, Lawyer, and Linguist, 1746-1794, Oxford, Oxford University Press, 2011, p. 75.

7. En 1780, elle épouse Henry Savile de Starck Esq dont les Mémoires sont une source importante d'informations à son sujet (Claude Pillement, Mademoiselle de Fauque, alias Marianne Agnès Pillement 1720-1804. Notice biographique chronologique. La femme, sa vie, son œuvre, Paris, Éditions Visages du monde, 2008, p. 31-32).

8. Raymonde Robert, "Mlle de Falques », dans Huguette Krief et Valérie André (dir.), Dictionnaire des femmes des Lumières, Paris, Honoré Champion, 2015, p. $449-45^{2}$.

9. Ibid., p. 451.

10. Mlle de Falques, Histoire de Madame la marquise de Pompadour, Londres, S. Hooper, 1759, p. 3. Exemplaire de la Bibliothèque d'État de Bavière. 
l'époque ${ }^{11}$ » a fait l'objet de nombreuses tentatives pour être rachetée et détruite au moment de sa publication ${ }^{12}$. Parce qu'il a tous les traits d'un roman, ce texte, répertorié dans la Bibliographie du genre romanesque français, 1751-1800 $(1977)^{13}$, de même que dans la liste de romans et d'histoires à propos des Anglais que compile Josephine Grieder en appendice dans Anglomania in France, 1740-1789 $(1985)^{14}$, est considéré comme tel dans le cadre de la présente étude. Alors qu'en 1879, les bibliographes concluaient à une pseudo-traduction ${ }^{15}$, la version française publiée pour la première fois en 1759 est effectivement précédée d'une version anglaise publiée en 1758 de la main de la même auteure ${ }^{16}$. Publié d'abord en anglais, puis en français et ensuite en allemand, le texte est réédité une douzaine de fois jusqu'en 1770, puis un siècle plus tard en 1879. Il faut également noter que les Mémoires pour servir à l'histoire de la marquise de Pompadour traduits de l'anglais sont publiés sans nom d'auteur en 1763 à Londres chez S. Hooper. Bien que cet ouvrage soit parfois attribué à Ange Goudar ${ }^{17}$, le topos de la pseudo-

11. Mark Brownell, Monsieur d'Eon is a Woman, Toronto, Playwrights Union of Canada, 1999, p. 162. Je traduis.

12. L. F., "Notice», dans Histoire de la marquise de Pompadour par Mlle de Fauques réimprimée d'après l'édition originale de 1759 avec une notice sur le livre et son auteur, Paris, Le Moniteur du bibliophile, 1879, p. 10-12.

13. Angus Martin, Vivienne G. Mylne et Richard Frautschi, Bibliographie du genre romanesque français 1751-1800, Mansell, Londres, 1977, p. 58, entrée 59.12.

14. Josephine Grieder, Anglomania in France, 1740-1789: Fact, Fiction, and Political Discourse, Genève, Droz, 1985, p. 154.

15. Dans sa notice, L. F. affirme que «tous les bibliographes s'accordent à considérer cette traduction comme supposée et à tenir pour originale la version française » (Histoire de la marquise de Pompadour par Mlle de Fauques, op. cit., p. 9).

16. Le World Catalogue précise bien que Mlle de Falques est l'auteure du roman The History of the Marchioness de Pompadour publié en 1758. La Bibliographie du genre romanesque français 1751-18oo comporte la note suivante: «Une version anglaise existe, mais d'après des indications dans le texte, il est clair que cette version est une traduction du texte français. Il se peut néanmoins que la traduction ait été publiée avant l'Histoire, pour appuyer la notion que l'ouvrage était d'origine anglaise» (op. cit., p. 58). Il faut noter qu'une grande conformité lie les versions anglaise et française. La pseudo-traduction en tant que stratégie de légitimation connaît donc sous la plume de Mlle de Falques un traitement des plus élaborés: le texte initialement rédigé en français aurait été traduit en anglais afin d'être publié d'abord dans cette langue pour assurer que sa version française publiée un an plus tard, qui constitue l'original, apparaisse comme une traduction légitime. Voilà à quelles précautions la romancière fait appel!

17. Claude Cristin et Marie-Françoise Luna, «Pierre Ange Goudar», dans Dictionnaire des journalistes 1600-1789, Oxford, Voltaire Foundation, http://dictionnaire-journalistes.gazettesı8e.fr/journaliste/351-pierre-ange-goudar. 
traduction, de même que le choix de l'éditeur laissent penser qu'il pourrait s'agir d'une suite de la main de Mlle de Falques. D'ailleurs, l'«Avis de l'éditeur» précise que l'auteur(e) se considère comme le «simple continuateur des mentionnées petites parties premières ${ }^{18}$ ». De plus, il faut préciser que Mlle de Falques poursuit son travail à visée biographique en 1766 dans The Life of the Marchioness de Pompadour. The Fourth edition revised and enlarged with a continuation from 1757 to her death, ouvrage qu'elle publie chez S. Hooper. Marie-Laure Girou Swiderski affirme que vingt ans après sa première publication, le roman Histoire de Madame la marquise de Pompadour traduite de l'anglais continue de circuler clandestinement ${ }^{19}$. Aux yeux de Voltaire, «la moitié de l'ouvrage est un tissu de calomnies, mais ce qu'il y a de vrai fera passer ce qu'il y a de faux à la postéritée ${ }^{20}$ ». Pour sa part, Grimm n'hésite pas à dénigrer le texte dans sa Correspondance littéraire où il écrit que nous devons à Mlle de Falques de "mauvais romans dans lesquels les sots disent qu'il y a de l'esprit ${ }^{21} »$. Seule Fortunée Briquet reconnaît le mérite littéraire de la romancière dans son Dictionnaire historique, littéraire et bibliographique des Françaises $(1804)^{22}$. L'Histoire de Madame la marquise de Pompadour propose une peinture d'une figure historique légendaire qui subjugue mari, amant et roi grâce, entre autres, aux objets de luxe, à la mode et aux pratiques ostentatoires qui règnent à la cour. Quelle publicité fait ce roman au

18. "Avis de l'éditeur", dans Mémoires pour servir à l'histoire de la marquise de Pompadour, Londres, S. Hooper, 1763 , p. 6.

19. Marie-Laure Girou Swiderski précise qu’il figure «parmi les livres interdits les plus demandés aux colporteurs et aux libraires clandestins jusqu’à la veille de la Révolution » ( L'écriture subversive de Mademoiselle de Fauques: La dernière guerre des bêtes et l'Histoire de Mme la marquise de Pompadour ", dans Marie-Laure Girou Swiderski (dir.), Terrce incognitce de l'écriture féminine, Ottawa, Legas, 2004, p. 87.) Il faut toutefois noter que dans l'annexe des ouvrages les plus commandés que compile Robert Darnton dans son livre Édition et Sédition sont répertoriés les Mémoires de Mme la marquise de Pompadour et non pas l'Histoire de Madame la marquise de Pompadour (Robert Darnton, Édition et sédition. Lunivers de la littérature clandestine au XVIII siècle, Paris, Gallimard, 1991, p. 223 et 231).

20. «Lettre à M. Bertrand», citée dans la notice de L. F. (Histoire de la marquise de Pompadour par Mlle de Fauques, op. cit., p. 14).

21. Friedrich Melchior Grimm, Correspondance littéraire, philosophique et critique, 15 juin 1755, dans Maurice Tourneux, Grimm, Diderot, Raynal, Meister, etc., Paris, Garnier, 1878 , t. III, p. 43.

22. Fortunée Briquet, «Fauque (Mademoiselle de)», dans Dictionnaire historique, littéraire et bibliographique des Françaises, Paris, de Gillé, 1804, p. 142-143. 
luxe et à son règne en France? De quelles manières la représentation de la cour de Louis XV expose-t-elle le pouvoir que concèdent le luxe et ses objets?

Depuis la publication de La Fable des abeilles (1705) de Mandeville, savants et littéraires s'interrogent sur les effets sociaux du luxe. Ce texte qui n'est traduit en français qu'en 1740 inspire Voltaire, Montesquieu et Rousseau et donne le coup d'envoi au débat sur le luxe qui façonne l'avenir de la France. Selon Dominique Margairaz, ce dernier constitue «le lieu conflictuel d'affrontement de représentations concurrentes de la société, de ses fondements et de son devenir ${ }^{23} »$. Le luxe trouve chez Jean-François Melon, David Hume et G.-M. Butel-Dumont ses plus grands défenseurs ${ }^{24}$. Les concours académiques sur la richesse, le progrès, le commerce, l'industrie, la vanité, l'inégalité, etc. confirment la préoccupation grandissante que suscite ce sujet controversé. Bien qu'à l'époque de Louis XV le luxe devienne un agent de nivellement social, comme le constate Mme de Genlis dans son Dictionnaire critique et raisonné des étiquettes de la cour (1818) où elle pose un regard nostalgique sur la France d’Ancien Régime et se désole que "presque toutes les fortunes peuvent y atteindre; et qu'ainsi il a contribué à confondre tous les états, toutes les classes, et à ruiner toutes les familles ${ }^{25}$ ", il continue de s'imposer comme moyen d'usurpation, mais surtout de contrôle. Le luxe tire tout son sens des pratiques et des usages qu'en fait la société, de sorte qu'il se conçoit comme une « construction sociale qui n’a de sens qu'en référence à une hiérarchie des individus et de leurs besoins ${ }^{26}$ ». Voilà ce que sait parfaitement l'héroïne de Mlle de Falques, savoir qu'elle utilise à son avantage pour défendre sa place dans un monde de spectacle où briller, c'est exister. Pour la maîtresse du roi, le luxe devient un allié important, voire une

23. Dominique Margairaz, «La querelle du luxe au XviıI siècle», dans Jacques Marseille (dir.), Le luxe en France du siècle des Lumières à nos jours, Paris, Association pour le développement de l'histoire économique (ADHE), 1999, p. 25.

24. Voir l'Essai politique sur le commerce (1734) de Jean-François Melon, l'Essai sur le luxe (1752) de David Hume et la Théorie du luxe (1771) de G.-M. Butel-Dumont.

25. Mme de Genlis, Dictionnaire critique et raisonné des étiquettes de la Cour ou l'esprit des étiquettes et des usages anciens, Paris, P. Mongie Aîné, 1818, t. I, p. 334.

26. Jean-Yves Grenier, «De la richesse à la valeur: les métamorphoses d'une notion au XVIII ${ }^{\mathrm{e}}$ siècle», dans Jacques Berchtold et Michel Porret (dir.), Être riche au siècle de Voltaire, Genève, Droz, 1996, p. 27. 
« exigence absolue $e^{27}$ », pour conserver sa place. De la même façon qu'il sert de «moyen d'auto-affirmation sociale ${ }^{28}$ » à la noblesse, le luxe se présente à ses yeux comme un instrument de pouvoir privilégié. Si l'on considère qu'à l'époque des Lumières, «l'ambition, le désir de se démarquer, l'ostentation et la soif de pouvoir motivent le luxe» et que l'envie de paraître supérieur se réalise grâce au «luxe quantitatif ${ }^{29}$ ", la conduite de la Marquise de Pompadour dans le roman de Mlle de Falques ne fait pas exception et s'inscrit dans la foulée des pratiques et des usages sociaux établis. Depuis le règne de Louis XIV, faste, superflu, splendeur et excès sont les mots d'ordre à la cour où le luxe constitue un dispositif efficace pour étendre son pouvoir. Miroir aux alouettes, il constitue un leurre nécessaire. Au cœur de ce «jeu de montre ${ }^{30} »$ se lit l'ambition d'atteindre un statut toujours plus brillant.

Parce qu'elle maîtrise parfaitement l'art de plaire, la favorite que met en scène Mlle de Falques règne en souveraine à la cour. Libérée de son époux par la protection du roi qui n’hésite pas à l'exiler un an en Avignon grâce à une lettre de cachet $^{31}$, elle jouit d'une indépendance et d'un pouvoir de décision inégalés. En dépit de la haine du peuple et de ses ennemis, elle réussit à conserver son ascendant sur le roi pendant au moins quinze ans ${ }^{32}$. L'influence et le pouvoir que s'approprie Mme de Pompadour, tributaires en grande partie de la « représentation $^{33}$ » dont les nobles réclament le monopole, font d'elle un des personnages féminins qui marque l'histoire littéraire par ce que Judith

27. Renatto Galliani, Rousseau, le luxe et l'idéologie nobiliaire. Étude sociohistorique, Oxford, Voltaire Foundation, coll. «SVEC», 268, 1989, p. 129.

28. Id.

29. Werner Sombart, Luxury and Capitalism, Ann Arbor, University of Michigan Press, 1967 , p. 61. Je traduis.

30. Philippe Perrot, Le luxe. Une richesse entre faste et confort, XVIII ${ }^{e}$-XIX ${ }^{e}$ siècle, Paris, Seuil, 1995, p. 51.

31. «Le pauvre d'Estiolles abandonné ainsi de son épouse, jetta [sic] les hauts cris et remplit le monde de ses plaintes. Il alloit tout tenter pour la faire revenir à son devoir, lorsqu'il reçut une lettre de cachet qui le reléguoit à Avignon » (Mlle de Falques, Histoire de Madame la marquise de Pompadour, op. cit., p. 28).

32. À la fin du roman, l'instance narrative signale qu' « [i]l y a quinze ans qu'elle règne en qualité de Maitresse du Roi » (ibid., p. 157).

33. "Because représentation served to produce social distinctions and a hierarchy of power, it was supposed to be a monopoly of the governing ranks » (John Shovlin, «The Cultural Politics of Luxury in Eighteenth-Century France », French Historical Studies, 23, 4, 2000, p. 583). 
Butler appelle la «capacité d'agir ${ }^{34} »$. En effet, elle assume pleinement son existence, elle devient l'arbitre par excellence du goût à la cour et la cause de décisions politiques et culturelles inusitées.

Son pouvoir sur le roi s'enrichit d'une perte dont l'amour s'accommode difficilement, soit la possibilité d'entretenir des relations sexuelles. L'instance narrative confirme que «sa puissance a été assurée par l'événement même, qui devait la détruire, [...] par la cessation d'un commerce de volupté sensueller [sic] entre elle et le Roi ${ }^{35}$ ». Non seulement elle maintient son empire sur le roi, mais elle s'arroge aussi un droit de regard et un pouvoir de décision sur ses relations sexuelles en lui procurant un asile où se livrer à l'amour sans dangers. Le lieu qu'elle met à sa disposition, son ermitage, pourvu de tous les «embelissément $[\mathrm{s}][$ sic $]$ essentiel[s] » où le choix des tableaux et des meubles, la décoration des chambres, la disposition des jardins, les fleurs qui ornent les plates-bandes, les terrasses et les allées, bref le «goût exquis ${ }^{36}$ » qui y règne rappelle indéniablement sa présence et empêche le roi d'oublier entièrement son souvenir. Une telle faveur est généreusement payée de retour par un amant sensible à «l'obligation de réparer quelque tort qu'il lui auroit fait ${ }^{37} »$. Femme de pouvoir, elle est comparée à Mme de Maintenon : «le monde aura un second Tome de Madame de Maintenon ${ }^{38} »$. À la différence de celle-ci, qui établit à la cour une ère de dévotion et d'austérité, Mme de Pompadour célèbre plutôt le plaisir et le divertissement dont elle «ordonne tout ${ }^{39}$ ». Telle «l'âme [des] fréquents et dispendieux voiages $[\text { sic }]^{40}$ » du roi, elle joue un rôle essentiel à la cour où elle figure en «oracle ${ }^{41}$ ». Ultime arbitre du goût, «tout est soumis à ses caprices: tout est réglé par ses volontés ${ }^{42}$ », de sorte que le luxe connaît une nouvelle étendue et que la frivolité l'emporte : «On joue gros jeu; on chasse; on rit. On perd dans

34. Judith Butler, Trouble dans le genre. Pour un féminisme de la subversion, préface d'Eric Fassin, traduction de Cynthia Kraus, Paris, La Découverte, 2005.

35. Mlle de Falques, Histoire de Madame la marquise de Pompadour, op. cit., p. 137.

36. Ibid., p. 87.

37. Ibid., p. 91.

38. Ibid., p. 147.

39. Ibid., p. 149.

4o. Id.

41. Ibid., p. $3^{8 .}$

42. Ibid., p. 149. 
un dédale de distractions, le goût et la faculté de penser à des choses sérieuses. On néglige les affaires, qui ne figurent plus qu'en second ${ }^{43}$ ». Issue de la bourgeoisie, mais plus précisément d'une relation adultère ${ }^{44}$, elle parvient à régler le mode de vie de la noblesse à la cour ; pécheresse car épouse infidèle, elle se targue d'être la maîtresse du roi; femme, elle se fait homme d'état: «elle afecte [sic] un personage [sic] encore plus grand et plus élévé [sic]. Elle veut trancher du despote et donner à la machine politique, le mouvement qui lui plait ${ }^{45}{ }$. Sous la plume de Mlle de Falques, Mme de Pompadour fait céder les préjugés de sexe et de rang qui perdurent à l'époque des Lumières et transgresse toutes les limites qu'impose l'ordre établi. Par quels moyens réalise-telle son ouvrage? La ruse se présente comme le moteur de ses actions. Enfant de l'usurpation, le luxe l'autorise, voire l'appelle. Dès le début du roman, le vêtement est présenté sous les traits d'un instrument de conquête privilégié: «Elle se fit faire un habit dans ce goût fin et exquis [...] et tout fut préparé pour atteindre le but qu'elle s'étoit fixét6 $»$. Aux yeux de la spectatrice qui cherche surtout à être vue et à fixer le regard du roi lors de ses parties de chasse, l'habit se présente comme un acteur important dans la quête de séduction. Son habileté à distraire le roi ou plutôt à lui faire "passer le tems ${ }^{47}$ » et à vaincre l'ennui la distingue de ses consœurs. Certes, pour remplir cet objectif avec succès, la dépense s'avère nécessaire.

Au cours du siècle, penseurs et philosophes s'efforcent de distinguer différents types de luxe: celui d'ostentation, de magnificence, de vanité, de mollesse, etc. Bien qu'il soit le plus souvent considéré comme futile et frivole, il figure néanmoins comme un passage obligé à la cour. LEncyclopédie précise que le danger réside dans l'abus et l'excès et non pas dans le luxe en lui-même, qui après tout apparaît comme un «bien ${ }^{48}$ ». Aux yeux de l'héroïne de Mlle de Falques, l'excès constitue un moyen efficace pour conserver sa place à la cour.

43. Ibid., p. 149 .

44. «Pendant l'éloignement de son mari, [Mme Poisson] accoucha d'une fille qui est aujourd'hui la fameuse Madame de Pompadour. Quelque compte qu'elle eut voulu faire, il ne lui auroit pas été possible de faire passer cet enfant pour un ouvrage de son mari ( (ibid., p. 5).

45. Ibid., p. 152.

46. Ibid., p. 11.

47. Ibid., p. 31.

48. Saint-Lambert, «Luxe», art. cit. 
L'habitude du luxe risque de désensibiliser l'être humain et de l'empêcher de reconnaître la vraie grandeur et la beauté, comme le prévient Saint-Lambert dans l'Encyclopédie ${ }^{49}$. Au contraire, pour Mme de Pompadour, l'habitude la protège contre la rivalité féminine et préserve le roi des charmes d'autres femmes: le roi «ne sentoit plus les impressions de la pure nature, parce qu'il avoit vécu trop longtems sous le charme de l'art, charme qu'il sembloit ètre [sic] hors d'état de rompre $^{50} »$. Mme de Pompadour trouve donc tous les moyens d'éviter les pièges qui menacent le luxe et réussit au contraire à en tirer profit.

À une époque où la consommation atteint un nouveau seuil ${ }^{51}$ et où le progrès matériel de la France connaît un certain nombre d'avancées, la «fonctionnalitéén ${ }^{22}$ ou la «commodité53 ${ }^{3}$ d'objets pratiques susceptibles de procurer un plus grand confort transforment la culture matérielle. Ainsi, lorsque Mme de Pompadour s'entiche de «[c]olifichets, [de] nouvelles inventions de modes, [de] bimblotage [sic], [de] bijoux, [de] décorations de chambres pour les diférentes [sic] saisons de l'année, [de] meubles dans un goût nouveau [...], [de] couteuses babioles ${ }^{54}$ ", elle témoigne moins de l'émulation qui gouvernait la relation au luxe à l'époque de Louis XIV que du plaisir personnel. En 1771, G.-M. Butel-Dumont, un des plus importants défenseurs du luxe, constate effectivement que la relation aux objets a évolué vers une tendance à «augmenter l'expérience sensuelle du monde et [à] améliorer ainsi son état mental"55 ». Grâce à son «imagination

49. Ibid.

50. Mlle de Falques, Histoire de Madame la marquise de Pompadour, op. cit., p. 91.

51. "From 1650 to 1800 , elites, the middling sort, and, to some degree, the laboring classes began to consume goods on a previously unthinkable scale, particularly clothing, home furnishings, and exotic colonial products. Everything from kitchenware to underwear, tables to tea sets, and wigs to watches began to circulate as never before, irrevocably changing the relationship between people and things » (Michael Kwass, "Between Words and Things: "La Querelle du luxe" in the Eighteenth Century», MLN, 130, 4, 2015, p. 772).

52. Philippe Perrot, Le luxe, op. cit., p. 66.

53. Voir la Théorie du luxe (1771) de G.-M. Butel-Dumont.

54. Mlle de Falques, Histoire de Madame la marquise de Pompadour, op. cit., p. 122.

55. G.-M. Butel-Dumont cité dans Michael Kwass, «Between Words and Things: "La Querelle du luxe" in the Eighteenth Century», art. cit., p. 781. Il faut également noter que «l'idée d'un pouvoir d'éveil et d'un pouvoir d'influence des objets [...] sur l'individu est une donnée fondamentale de la psychologie sensualiste», comme l'affirment Christophe Martin et Catherine Ramond («Présentation», dans 
extraordinaire ${ }^{56} »$, Mme de Pompadour invente de nouvelles magnificences: au milieu de l'hiver, elle dispose des fleurs de porcelaine arrosées de parfum dans des pots, ce qui plaît fort au roi, stupéfié par l'illusion. Les courtisans concluent qu'«il n'étoit rien dans la nature ni hors d'elle, que Madame de Pompadour ne vint à bout de soumettre au despotisme de son art $^{57} »$. En s'assurant la mainmise sur le progrès matériel, elle étend son pouvoir aux objets qui la secondent dans sa quête. Parce qu'ils sont le lieu d'une véritable performance de la consommation et de la possession ${ }^{58}$, les meubles et les vêtements façonnent et codifient les mouvements corporels, l'apparence et la conduite des courtisans. Les objets de luxe qu'acquiert l'héroïne se lisent donc comme des instruments puissants qui en disent long sur l'empire socioculturel qu'elle se construit. Fenêtre sur la sociabilité à la cour, ils traduisent sa quête de pouvoir et conditionnent son identité et sa réputation. En choisissant des décorations de chambre selon les saisons de l'année et des meubles nouveaux, l'héroïne transforme et renouvelle les paramètres de sociabilité mondaine établis et lance un véritable défi à son entourage. En jetant les bases de nouveaux usages, Mme de Pompadour décide de ce qu'on pourrait appeler le «jeu des objets». Elle s'impose par ses habitudes de consommation qui, toutes personnelles soient-elles, n'en demeurent pas moins publiques, de sorte qu'elles font la publicité à la fois d'une nouvelle relation aux objets et de la richesse dont elle jouit et qu'elle est libre de rediriger selon sa volonté. En effet, Mme de Pompadour obtient à son époux l'occasion d'acheter à prix réduit l'une des plus belles maisons de Paris dont l'ameublement était «magnifique », où tout était «de vieux lacq [sic]», où le «goût régnait», où «les murailles et les portes étaient plaquées de fines glaces de miroir » et où les «intervalles en étoient couverts par des guirlandes de fleurs, peintes sur le verre, de la main des plus habiles

Christophe Martin et Catherine Ramond (dir.), Esthétique et poétique de l'objet au XVIII siècle, Bordeaux, Presses universitaires de Bordeaux, 2005, p. 11).

56. Mlle de Falques, Histoire de Madame la marquise de Pompadour, op. cit., p. 123.

57. Id.

58. Mimi Hellman précise bien que «the practice of consumption was visual and kinetic; objects were not simply owned, but indeed performed» («Furniture, Sociability, and the Work of Leisure in Eighteenth-Century France », Eighteenth-Century Studies, 32, 4, 1999, p. 417). Plus loin, l'auteure convient que « objects were exemplary social actors» (p. 436). 
maitres de Paris $^{59} »$. Elle agit ainsi non pas parce qu'elle se soucie de son bien-être et encore moins de devoir racheter sa faute auprès de lui, mais bien parce qu'elle désire rappeler son rang à son entourage. Étendard de son statut, la magnificence de la maison de son époux est une preuve de sa propre importance à la cour. C'est pourquoi elle n'hésite pas à l'enrichir ni à lui choisir une maîtresse d'un certain rang, stratégies utiles pour asseoir sa réputation et confirmer son ascension sociale. La pension qu'elle oblige son époux à verser à sa maîtresse ${ }^{60}$ s'explique de la même façon. Pour la même raison, elle obtient à son frère, qui n'est «digne d'attention que parce qu'il étoit son frère ${ }^{61}$ », le titre de Marquis de Vandière et le poste de Directeur des bâtiments, jardins, arts et manufactures du roi.

Au service de sa quête de pouvoir, le luxe prolifère sous la forme de cadeaux. Parce qu'elle réussit à faire reconnaître au roi sa valeur propre, la Marquise de Pompadour s'assure le plus grand dévouement: «L'impression de ce qu'il lui devoit, étoit si forte, que rien ne lui coutoit trop $\operatorname{cher}^{62}$ ». Le roi qui, grâce à elle, acquiert «le goût de donner ${ }^{63}$ » ne compte plus les dépenses. Voilà un marché sûr de contribuer à son bien-être matériel. En effet, «un présent en amenoit un autre et le dernier n'étoit que le garant de celui qui devoit le suivre ${ }^{64}{ }$. L'exemple de la Mailly, qui meurt dans un couvent sans le sou après que le roi a rompu avec elle, ou encore celui de Mme de Tournelle, qui est empoisonnée, ne suffisent-ils pas à justifier que la favorite dépense « impitoïablement $[\text { sic }]^{65}$ », étendant ainsi son pouvoir et assurant son avenir?

Preuves de son amour, les dons que lui fait le roi témoignent surtout de sa propre soumission et évitent d'obliger celle qui les reçoit. L'instance narrative considère d'ailleurs la situation du roi comme un «esclavage» et compare son attachement à de véritables « fers ${ }^{66}$ ». Sous la plume de Mlle de Falques, l'hérö̈ne échappe à la logique

59. Mlle de Falques, Histoire de Madame la marquise de Pompadour, op. cit., p. 131.

6o. «Il renvoia [sic] sa maitresse; mais il fut obligé de souscrire aux volontés de sa femme, en lui fésant [sic] une grosse pension» (ibid., p. 127).

61. Ibid., p. 35.

62. Ibid., p. 34 .

63. Ibid., p. 36.

64. Id.

65. Ibid., p. 37.

66. Ibid., p. 52. 
du «contre-don ${ }^{67} »$. Ailleurs dans le texte, le don fait contracter une dette à l'être à qui il est destiné et le contraint de respecter les conditions du pacte qu'il scelle. Par exemple, les biens matériels qu'obtient M. d'Estiolles en guise de réparation l'obligent à «laisser aller les choses comme elles alloient ${ }^{68} »$. Il en est de même pour l'époux de la jeune Murphy dont les «avantages assès $[$ sic $][. .$.$] considérables { }^{69}{ }$ " qu'il retire de son mariage l'engagent à assumer la paternité d'un enfant illégitime. Ressort principal d'une économie affective et symbolique, le don oblige, contraint, force et soumet celui ou celle qui le reçoit ${ }^{70}$, sauf dans le cas de Mme de Pompadour pour qui il est gratuit. Elle obtient un marquisat, qui lui vaut le titre de marquise de Pompadour, elle achète un palais, qu'elle fait reconstruire à la grande déception du peuple qui y voit un « vol fait au public ${ }^{71}$ ", elle reçoit en cadeau le château royal de Cressy, un des «biens de la Couronne ${ }^{72}$ », une maison de plaisance nommée Bellevue, dont la construction des jardins entraîne l'oppression de plusieurs propriétaires forcés «tyranniquement» de "céder leurs terres au prix qu'on voulut y mettre ${ }^{73}$ », et se voit octroyer un appartement des plus convoités à Versailles. Aux yeux de son amant, elle gagne en estime: «Plus la Pompadour recevoit, plus devenoit-elle

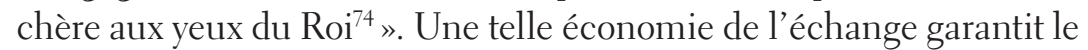
triomphe de la favorite. Les renvois qu'approuve Louis XV, le «trafic inoui ${ }^{75}$ » des charges qu'il autorise et les décisions qu'il prend pour punir les ennemis de sa maîtresse se lisent à leur tour comme des largesses. Le sort de M. de Resselier, condamné à la cage de fer au mont Saint-Michel pour avoir écrit un quatrain injurieux, témoigne des mesures que le roi est prêt à prendre pour défendre sa liaison; ni le supplice ni la torture ne sont exclus.

67. Sarah Horowitz, «Luxe, amour et transactions. La culture des bijoux sous l'Ancien Régime », Sociétés ङ Représentations, 38, 2, 2014, p. 136.

68. Mlle de Falques, Histoire de Madame la marquise de Pompadour, op. cit., p. 29.

69. Ibid., p. 94.

70. Marcel Mauss, «Essai sur le don », dans Sociologie et anthropologie, Paris, PUF, 1968 [1903], p. 147.

71. Mlle de Falques, Histoire de Madame la marquise de Pompadour, op. cit., p. $3^{8 .}$

72. Id.

73. Ibid., p. 59 .

74. Ibid., p. 41.

75. Ibid., p. 113. 
L'excès auquel Mme de Pompadour conduit le roi ou plutôt sa «grande passion à amasser des trésors ${ }^{76}$ » sont nécessaires à la sauvegarde de sa position, mais aussi à sa survie. C'est pourquoi elle tente d'acheter la principauté de Neufchâtel: «elle a pris la sage précaution de se ménager un asile assuré ${ }^{77}$ ", conclut l'instance narrative. Ainsi, tout excessive soit-elle, la dépense peut se révéler nécessaire, voire judicieuse. Il en est de même pour l'usage que fait la favorite de son influence. Au nombre des «abus » de sa position privilégiée, on compte «sa bénéficence $[$ sic $]$ envers les savans $[$ sic] », initiative qui, aux yeux de l'instance narrative, «mérite incontestablement les plus grands éloges $^{78}$ ». Parce qu'elle engage le roi à "récompenser le mérite ${ }^{79}$ ", ne contribue-t-elle pas à faire évoluer l'ordre établi dans une nouvelle direction? Favorable au développement des sciences et des arts, Mme de Pompadour pose des gestes concrets ${ }^{80}$ qui se révèlent d'une «utilité réelle ${ }^{81}$ » et qui servent la gloire du roi, mais aussi le progrès, valeur chère aux philosophes. Ainsi, à travers son emploi du luxe, Mme de Pompadour défend un certain nombre d'idéaux bourgeois qui triompheront au siècle suivant: individualisme, mérite, indépendance et utilitarisme ${ }^{82}$. En souscrivant à une «éthique du bonheur, propre à une conception rationaliste, relativiste, sensorielle et utilitaire du monde ${ }^{83}$ ", l'héroïne du roman de Mlle de Falques témoigne de l'évolution des idées qui marque le siècle des Lumières.

Lultime présent pouvant satisfaire Mme de Pompadour tient moins au luxe matériel qu'à l'honneur. En réclamant les «honneurs du Louvre » qui consistent à s'asseoir en présence de la reine et à en recevoir un baiser, Mme de Pompadour fait céder l'étiquette, «qui n’accorde guères cette prérogative qu'à des Duchesses », et la morale, puisqu'on y voit une grande «indiscrétion ${ }^{84} »$. Ce luxe, qui tient à la

76. Ibid., p. 73.

77. Ibid., p. 114. Je souligne.

78. Ibid., p. 119 .

79. Id.

80. Elle se rend dans les ateliers, y mène le roi, obtient des pensions, des logements et d'autres avantages aux plus méritants.

81. Mlle de Falques, Histoire de Madame la marquise de Pompadour, op. cit., p. 120.

82. Voir Renatto Galliani, «La société aristocratique et la société bourgeoise», dans Rousseau, le luxe et l'idéologie nobiliaire, op. cit., p. 226-235.

83. Ibid., p. 233.

84. Mlle de Falques, Histoire de Madame la marquise de Pompadour, op. cit., p. 61. 
distinction sociale, continue de se renouveler de la même façon que le faisait le luxe matériel. En effet, Mme de Pompadour devient «Dame du palais de la Reine ${ }^{85} »$, faveur rare et exceptionnelle accordée seulement à quelques femmes nobles ${ }^{86}$.

Aux yeux de l'instance narrative, le froid calcul aurait éteint l'amour dans le cœur de la favorite et formerait son plus grand défaut: «son cœur étoit sans pitié comme il étoit sans amour ${ }^{87}$ ». Elle n'aurait aimé ni le roi ni sa propre fille et serait parvenue à « conduire et [à] gouverner le Roi, par les aparences [sic] mêmes qu'elle s'en laisse gouverner ${ }^{88}$ ». L'ouvrage de la Marquise de Pompadour n'est donc pas le produit de la passion amoureuse et encore moins de l'amour maternel, mais d'un "génie supérieur ${ }^{89}$ » grâce auquel elle parvient à simuler et à feindre. L'instance narrative est catégorique: « tout étoit suposé [sic], tout emprunté. Son art étoit trop caché, pour qu'il put être découvert et manquer son coup. Sans paroitre jamais rien demander, elle obtenoit toujours tout ${ }^{90} »$. Si l'instance narrative lui reproche d'être avare, vaniteuse, insensible et orgueilleuse, elle reconnaît néanmoins les avantages que lui procurent la ruse et l'efficacité avec lesquelles elle mène son jeu: «il seroit à souhaiter, que pour le bonheur de l'humanité, son secret fut plus connu et son exemple plus suivi, qu'il ne l'est ${ }^{91}{ }^{»}$. Voilà que la satire cède sa place à l'éloge et que le pouvoir de la favorite du roi, désormais perçue comme un modèle à imiter, est encensé par la fiction.

Mme de Pompadour n'hésite pas à se tourner vers la mode pour affermir son capital culturel. Ce faisant, elle imite Louis XIV qui misait sur l'élégance et la splendeur vestimentaires pour normaliser les pratiques culturelles à la cour et pour étendre son pouvoir ${ }^{92}$. En

85. Ibid., p. 64.

86. Seules les femmes «les plus distinguées par leur naissance, par leur rang, et par leurs dignités » (id.) y sont admises.

87. Ibid., p. 40.

88. Ibid., p. 156.

89. Ibid., p. 39.

9o. Ibid., p. 39-40.

91. Ibid., p. 49. Je souligne.

92. Jennifer Jones précise que «Although Louis XIV never fully accepted fashion's imperious drive for novelty, throughout his long reign he attempted to capitalize on both the Frenchness of fashion and its theatricality " (Sexing La Mode: Gender, Fashion and Commercial Culture in Old Regime France, Oxford/New York, Berg, 2004, p. 19). 
donnant le ton, la marquise fixe les usages et les pratiques du goût français, véritable «geste unificateur ${ }^{93}{ }$. Elle «inventa un négligé, qui fut mis à la mode, sous le nom de robe à la Pompadour ${ }^{94}{ }^{\prime}$. Grâce à ce vêtement aguichant qui montre plus qu'il ne cache ses formes féminines, elle mise sur son pouvoir sensuel. Or, comme sa relation avec le roi est devenue platonique, il s'agit moins de séduire que d'imposer sa présence à la cour et dans le monde où le règne de la mode commence à se faire sentir ${ }^{95}$. D'ailleurs, l'instance narrative précise qu' «à la voir, on croioit $[s i c]$ lui entendre dire: Me voici ${ }^{96} »$. Preuve de l'influence qu'elle exerce à la cour, mais aussi en France, voire en Europe, le négligé si popularisé seconde son objectif. Pour sa part, le maquillage qu'elle utilise d'abord pour faire valoir sa beauté naturelle devient un véritable masque. Pour continuer de jouer le rôle qu'elle s'est donné, elle doit, en 1758, dissimuler son visage derrière « une couche de blanc et de rouge de l'épaissein $[$ sic $]$ d'un pouce ${ }^{97}$ ». Ainsi "plâtrée8 », son visage correspond parfaitement au modèle qu'elle a conçu malgré les empreintes que le temps y a laissées. Figure légendaire et éternelle, elle se présente à la fin du texte sous les traits d'une véritable chimère: sa

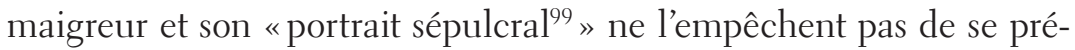
senter «au milieu de la grandeur, des richesses et de la faveur signalée du Roi, qu'elle a sû $[$ sic $]$ captiver $^{100} »$.

À une époque où la rivalité entre femmes est susceptible de changer spontanément le visage de la cour, le règne de Mme de Pompadour bénéficie paradoxalement de la solidarité féminine dans le roman de Mlle de Falques. Au sein du marché des apparences qu'elle conclut, la

93. Ina McCabe utilise cette expression pour désigner l'effet que produisent les efforts de Louis XIV : «The new category of "Frenchness" crafted by Louis extended across classes and was a unifying gesture in which clothing marked participation in the nation" (Orientalism in Early Modern France: Eurasian Trade, Exoticism, and the Ancien Régime, Oxford/New York, Berg, 2008, p. 269). p. 158 .

94. Mlle de Falques, Histoire de Madame la marquise de Pompadour, op. cit.,

95. Joan Dejean précise qu'à compter du règne de Louis XIV, les femmes dépensent trois fois plus que les hommes pour se vêtir et que la mode devient de plus en plus genrée (The Essence of Style, op. cit., p. 40).

96. Mlle de Falques, Histoire de Madame la marquise de Pompadour, op. cit., p. 159.

97. Id.

98. Id.

99. Ibid., p. 160.

100. Id. 
maîtresse qu'elle choisit à son époux, Mme de la Mothe, joue un rôle important. Si celle-ci bénéficie d'une pension considérable lorsqu’elle donne naissance à une fille illégitime, enfant dont Mme de Pompadour n'hésite pas à avoir « tout le soin imaginable ${ }^{101}$ », en retour, son rang et sa collaboration permettent à Mme de Pompdaour d'atteindre les objectifs qu'elle affectionne. Même l'instance narrative, qui pose un regard fort critique sur «le cœur embaumé de ruse ${ }^{102}$ » de Mme de Pompadour, exprime son admiration devant son parcours et estime que son exemple pourrait être utile à plus d'une femme: "Quelque grand que soit le danger auxquel [sic] les hommes seroient alors exposés; les femmes profiteroient infiniment [de son secret] et leurs desseins se verroient toujours courronnés [sic] par d'heureux succès ${ }^{103}$ ». Mme de Pompadour défend le droit à l'action, mais aussi le mérite personnel. Parvenue, elle incarne un nouveau modèle féminin qui ouvre des horizons inédits aux lectrices. En révélant son secret, qui consiste essentiellement à se conformer en tout à l'humeur du roi, l'instance narrative fait œuvre plus utile auprès des lectrices à qui elle enseigne une stratégie accessible à chacune puisque «[c]e n'est ni la grande beauté ni le grand esprit qui conduisent à ce but ${ }^{104}$ ». Tributaires d'une filiation féminine et maternelle, les leçons qu'assimile Mme de Pompadour proposent de nouvelles avenues pour conduire à la réalisation personnelle, sociale et matérielle. Pupille assidue dotée de dispositions naturelles, Mme de Pompadour comble les attentes de sa mère à un point tel que «[p]eut-être la trop grande joie qu'en eut [Mme Poisson] contribuat-elle [sic] à abréger ses jours ${ }^{105}$ ». En tant que personnage de fiction, Mme de Pompadour a tous les traits d'une femme de pouvoir qui a su combattre le feu par le feu en se taillant, grâce au luxe, une place de choix à la cour de Louis XV.

$\mathrm{Si}$ «le goût dominant des jouissances matérielles s'étend de Paris sur la France ${ }^{106}$ » à l'époque des Lumières, comme l'affirme Henri Baudrillart dans son Histoire du luxe (1880), la littérature poursuit sa

101. Ibid., p. 127.

102. Ibid., p. 160.

103. Ibid., p. 49. Je souligne.

104. Ibid., p. 50.

105. Id.

106. Henri Baudrillart, Histoire du luxe privé et public, Paris, Hachette et cie, 1880, vol. 4, p. 306. 
promotion pour qu'il s'étende à l'Europe tout entière. En se tournant vers la fiction pour porter un regard critique sur une figure historique importante, Mlle de Falques met en scène une héroïne qui reconnaît la valeur symbolique propre aux objets de luxe et à la mode, et qui, grâce à eux, se taille une place de choix. Le portrait qu'elle brosse de celle qui foule à ses pieds les «ministres les plus habiles, les plus grands Généraux de l'armée », qui «se roule dans des richesses immenses » et qui « dispos[e] à son gré [...] des trésors de l'État ${ }^{107}$ », en dépit du fait qu'elle est «tirée de la lie du peuple ${ }^{108}$ », montre que le luxe et ses objets sont des moyens utiles et efficaces pour s'affirmer, pour agir et pour conduire sa destinée. De fiction à libelle, le roman passe à l'Histoire ${ }^{109}$, témoignant par-là de ce que peut l'imaginaire du luxe. La fiction se comprend comme un espace où libérer le luxe de ses contraintes et renouveler le débat sur les dangers qui le guettent. Euvre audacieuse de la main d'une auteure dont la conduite n'a pas échappé à l'œil vigilant de la police ${ }^{110}$, l'Histoire de Madame la marquise de Pompadour donne à lire le pouvoir d'une femme à sa table d'écriture, mais aussi celui d'une femme à sa toilette. En effet, la publication, la traduction et les dizaines de rééditions de l'Histoire de Madame la marquise de Pompadour étendent le pouvoir de son hérö̈ne qui touche l'imaginaire des lecteurs et des lectrices de toute l'Europe.

107. Mlle de Falques, Histoire de Madame la marquise de Pompadour, op. cit., p. 112-113.

108. Ibid., p. 59.

109. Aux yeux des frères Goncourt, le roman de Mlle de Falques constitue une véritable biographie, voire «le seul corps d'histoire » qui porte sur la favorite (Edmond et Jules de Goncourt, Madame de Pompadour, Paris, Firmin Didot, 1888 [1881], p. 7). À son tour, l'ouvrage des frères Goncourt figure dans la bibliographie de biographies récentes sur Mme de Pompadour dont celle de Robert Muchembled, Mystérieuse Madame de Pompadour (2014), celle du Duc de Castries, La Pompadour (1983), et celle de Jean Nicolle, Madame de Pompadour et la société de son temps (1980).

110. Penny Arthur, «Le prix de la renommée littéraire: les notes de police concernant Mme de Graffigny, Mlle Falques et Mme Curé», dans Sébastien Côté et Sébastien Drouin (dir.), Surveillance, méfiance et art d'écrire dans les correspondances du long $18^{e}$ siècle, Oxford, Oxford University Studies in the Enlightenment, à paraître. 